\title{
Prévisions et rejeux des états de mer du globe à la plage
}

\author{
Rudy MAGNE ${ }^{1}$, Fabrice ARDHUIN ${ }^{1}$ \\ ${ }^{1}$ Service Hydrographique et Océanographique de la Marine, \\ Hydrographie, Océanogrpahie et Météorologie Militaire, \\ 13 rue du Chatellier, 29200 Brest, France \\ rudy.magne@shom.fr,fabrice.ardhuin@shom.fr
}

\section{Résumé :}

Le SHOM a développé un système de prévisions des états de mer fondé sur une approche multi-échelles, du global à la plage, combinant plusieurs modèles hauturiers de résolution relativement grossière et une série de loupes côtières. Ce système est mis en œuvre expérimentalement dans le cadre du projet Prévimer. Les applications sont diverses: de la conduite des opérations navales au génie côtier en passant par la télédétection ou l'océanographie. Les résultats hauturiers se révèlent de très bonne qualité, avec des erreurs de généralement entre 9 à $15 \%$ en rejeu pour $H_{m 0}$ et $T_{\mathrm{m} 02}$ moyennés sur 3 heures. A la côte, les états de mer calculés sont souvent moins précis, avec des erreurs atteignant $20 \%$ voire $30 \%$, en partie à cause d'une représentation insuffisante de la répartition angulaire de l'énergie de l'état de la mer au large.

\begin{abstract}
:
SHOM has developed a multi-scale wave forecasting system from the global ocean to the beach, combining several relatively coarse models and high resolution coastal zooms. This system is operated as part of the Previmer project, for multiple applications ranging from naval operations to coastal engineering, remote sensing and investigations of ocean circulation (drift, mixing ...). Offshore hindcasts prove to be very accurate with errors generally between 9 and $15 \%$ against in situ 3-hourly averaged values of $H_{m 0}$ and $T_{\mathrm{m} 02}$. In coastal areas, errors are typically larger, often exceeding $20 \%$. This increase in error is partly due to a poor modelling of the directional distribution of the offshore wave energy.
\end{abstract}

\section{Mots-clés :}

Vagues - Prévisions - Côtier - Rejeu - Validation

\section{Description du système actuel}

Le système de prévision des vagues mis en place par le SHOM est conçu pour

DOI: 10.5150/jngcgc.2008.036-M (disponible en ligne - http://www.paralia.fr - available online)


fournir les meilleurs estimations possibles de l'état de mer à toutes les échelles, $d u$ bassin océanique à la plage, en complément des systèmes mis en oeuvre par Météo-France. Ce système s'inscrit, entre autres, dans le cadre du projet interorganismes « Prévimer» (http://www.previmer.org). A ce titre, il est mis en œuvre sur le serveur de calcul "Caparmor» à Brest. Les applications visées sont multiples, depuis l'information $\mathrm{du}$ grand public jusqu'aux applications scientifiques et techniques, y compris la télédétection (analyses et corrections de biais de mesures), et le génie côtier. Le système est fondé sur une approche multiéchelles combinant plusieurs modèles hauturiers de résolution relativement grossière et une série de loupes côtières. Les modèles de grande emprise utilisent la version 3.14 du code WAVEWATCH III, développée en partenariat avec le service météorologique des Etats-Unis (NOAA/NCEP) et Météo-France (TOLMAN, 2007).

L'aspect «multi-grilles » est une nouveauté importante du code depuis la version 3.09 de WAVEWATCH III. Il s'agit d'une option de calcul qui permet un emboîtement de plusieurs domaines avec communication dans les deux sens. Cet option permet de réduire sensiblement le temps calcul, par exemple en désactivant les régions profondes ou loin des côtes dans les loupes côtières, ce qui peut réduire considérablement le nombre de nœuds de calcul. L'utilisation de cette option facilite aussi beaucoup la mise en place et la gestion de loupes, désormais gérées par un seul programme. Enfin, de nouvelles fonctionnalités comme le partitionnement de la du spectre d'état de mer sur l'ensemble de la grille de calcul, la prise en compte de la physique côtière (BATTJES \& JANSSEN, 1978; ARDHUIN \& MAGNE, 2007) et de nouveaux paramétrages de la génération et de la dissipation des vagues (BIDLOT et al., 2005; ARDHUIN et al., 2008a) sont également ajoutés dans cette version. Par ailleurs, de légères modifications ont été faites pour les paramètres de sortie (RASCLE et al., 2008) et les paramétrages des processus de génération et dissipation des vagues (ARDHUIN et al., 2008b). Cette version 3.14-SHOM, est ainsi mise en œuvre avec le paramétrage de BIDLOT et al. (2005), puis celui de ARDHUIN et al. (2008a) pour les prévisions «multi-grilles » et les rejeux («hindcast») réalisés après le 22 février 2008, et ARDHUIN et al. (2008b), après le 17 juillet 2008.

Au 17 juillet 2008, le système de prévision combine un modèle global de $0.5^{\circ}$ de résolution en latitude et longitude avec 2 modèles régionaux de $0.1^{\circ}$ de résolution, "Golfe de Gascogne-Manche », et «Méditerranée » (cf. figures 1 et 2, disponibles sur le site Prévimer), un modèle régional de l'arc Antillais à $0.05^{\circ}$ de résolution, et deux modèles côtiers sur la Manche centrale et la côte d'Azur avec 
des résolutions de $2 \mathrm{~km}$ et $500 \mathrm{~m}$ respectivement. Ces modèles sont exécutés deux fois par jour avec une échéance maximale de 6 jours, forcés par les prévisions du Centre Européen de Prévisions Météorologiques à Moyen Terme (CEPMMT / ECMWF) (résolution nominale de $0.5^{\circ}$, toutes les 6 heures, et $0.25^{\circ}$ toutes les $3 \mathrm{~h}$ pour les prévisions ultérieures au 15 juin 2008). L'ensemble de ce système nécessite 45 minutes de calcul sur 64 unités de calculs (16 nœuds quadri-cores).

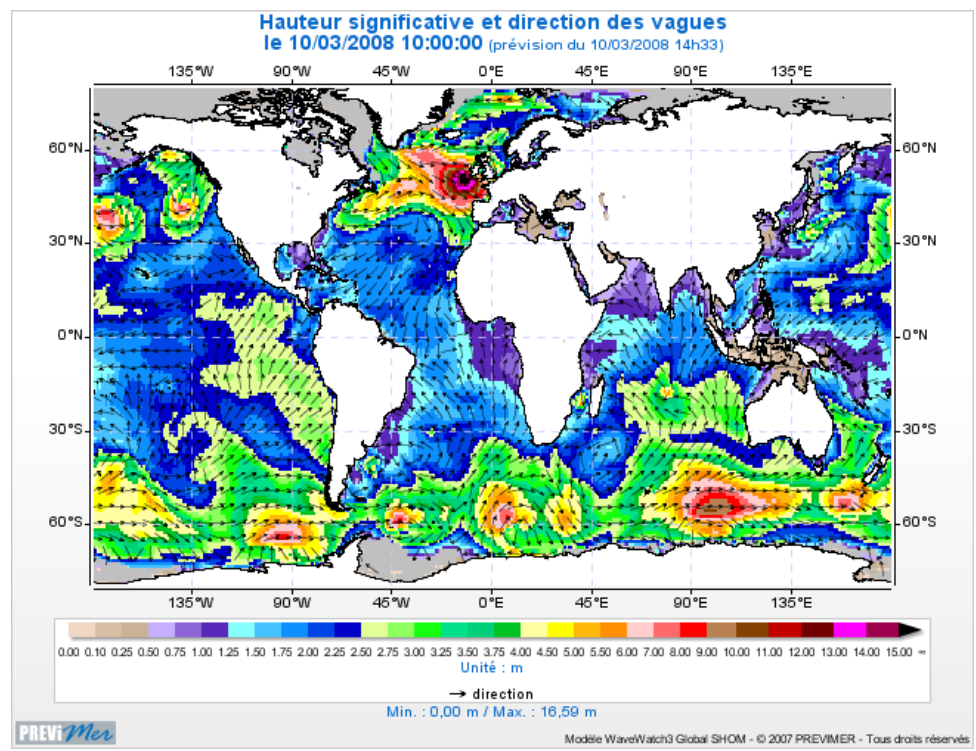

Figure 1. Modèle Global 0.5: $H_{m 0}$ calculé pour le 10/03/2008 à $9 \mathrm{~h}$ UTC avec les vents prévus du cycle de 00h UTC du 10/03/2008.
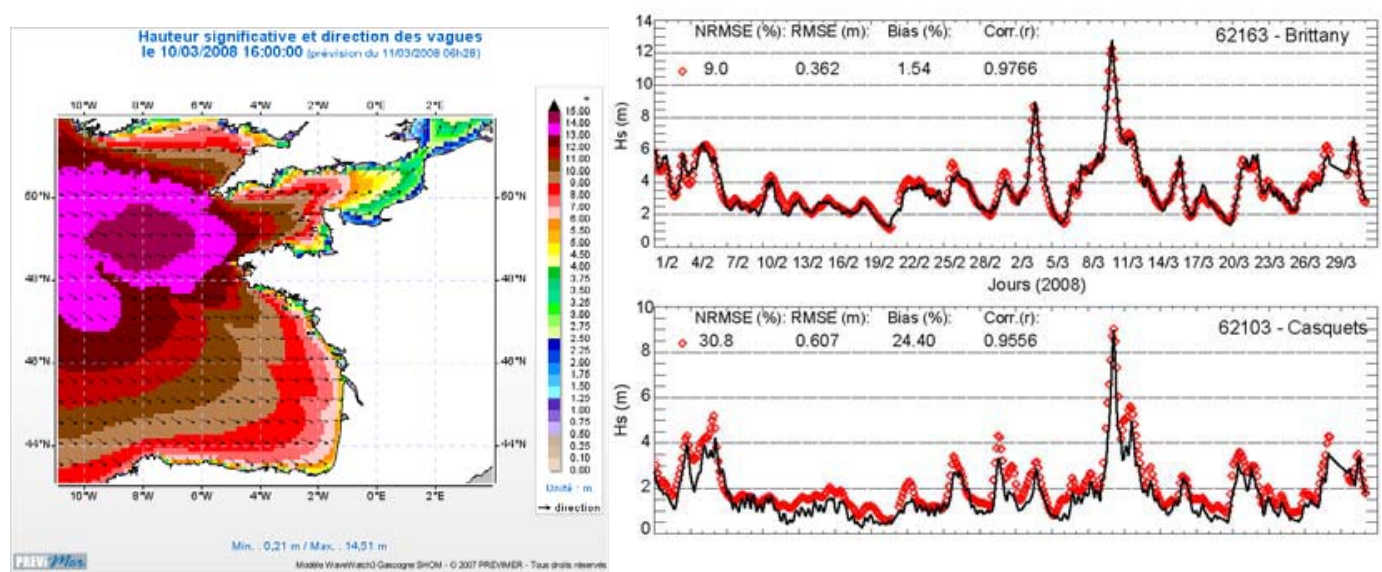

Figure 2. Modèle Gascogne-Manche 0.1 ${ }^{\circ}: H_{m 0}$ calculé le 10/03/2008 et statistiques pour les analyses réalisées en février et mars 2008 dans le Golfe de Gascogne et en Manche.

En zone côtière le système utilise un code parabolique de réfraction-diffraction (KIRBY, 1986) sur une bathymétrie de résolution 10 à $300 \mathrm{~m}$. La mise en oeuvre de ces calculs est très rapide (moins de 5 minutes sur un seul processeur pour 
l'ensemble de la chaîne Prévimer), car les propagations sont précalculées pour un niveau d'eau moyen et sans courant. La précision des résultats est souvent supérieure à celle modèles plus complets de génération de vagues comme SWAN par exemple qui souffre de schémas numériques très diffusifs pour le calcul de la réfraction, et utilise des limiteurs qui permettent une convergence rapide $d u$ calcul, mais biaisent le résultat.

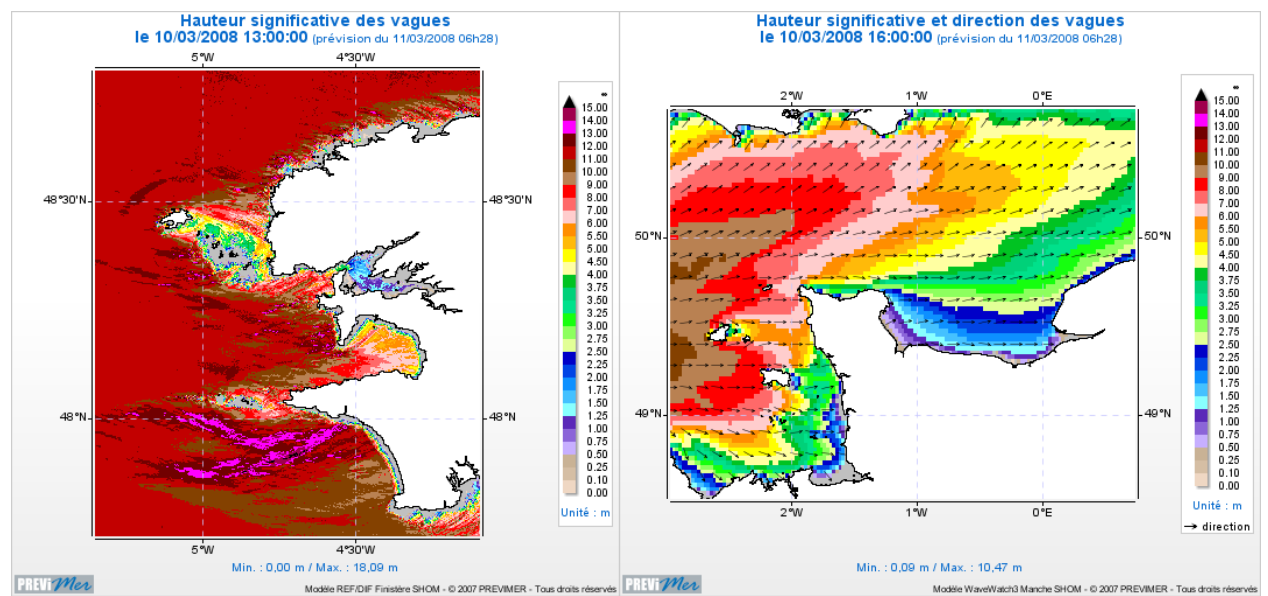

Figure 3. Modèles Iroise (Ref-Dif) et Manche(WAVEWATCH III), $H_{m 0}$ calculés pour le 10/02/2008

\section{$2 \quad$ Validation}

\subsection{Modèles hauturiers}

Pour assurer la qualité et l'amélioration des résultas, le SHOM participe au programme de comparaison de modèles du JCOMM (The Joint WMO-IOC Technical Commission on Oceanography and Marine Meteorology), avec une validation mensuelle sur plus de 250 points de mesure in situ dans le monde (BIDLOT et al., 2007). Pour pallier à la couverture spatiale limitée des mesures in situ, concentrées essentiellement dans l'hémisphère nord, et le long des côtes, des comparaisons avec les altimètres Jason, ENVISAT et GFO ont également été réalisées en collaboration avec l'Ifremer (RASCLE et al., 2008). Par ailleurs le paramétrage de la dissipation de la houle a été déterminé à partir de partitions de houle (hauteur, période et direction) déduites du mode vague du radar à synthèse d'ouverture du satellite ENVISAT (traitement réalisé par la société CLS). 


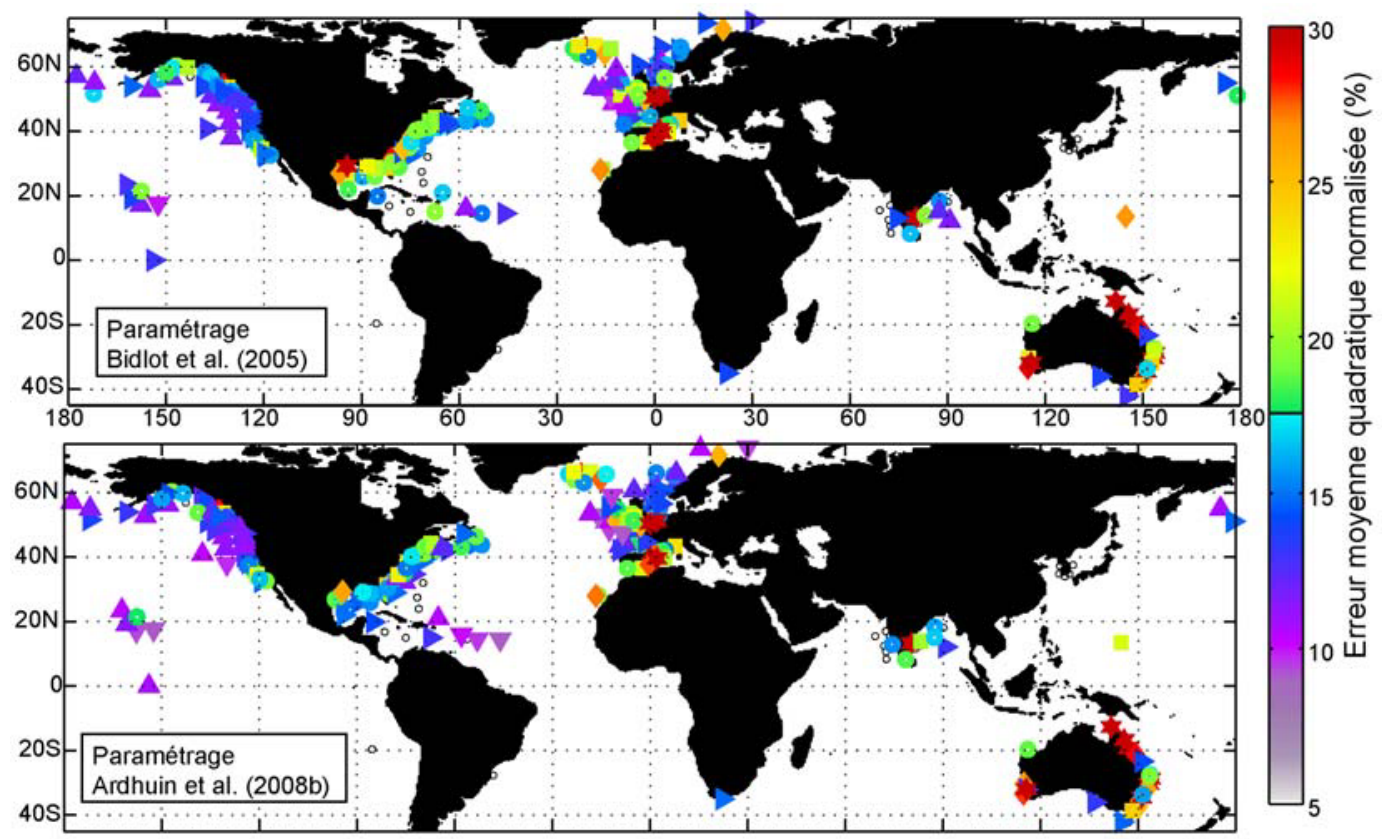

Figure 4: Carte des erreurs moyennes quadratiques normalisées par la moyenne des observations (NRMSE) en pourcents (en couleurs), sur tous les points de mesure JCOMM, pour l'année 2007. En haut : résultat avec le paramétrage de BIDLOT et al. (2005), en bas avec celui de ARDHUIN et al. (2008). Pour faciliter la lecture des couleurs, les valeurs $x$ dans les gammes $x<10 \%, 10<x<12.5 \%$, $12.5<x<15 \%, 15<x<20 \%, 20<x<25 \%, 25<x<30 \%$ et au-dessus de $30 \%$ sont représentées par les symboles $\boldsymbol{\nabla}, \boldsymbol{\Delta}, \boldsymbol{\bullet}, \bullet, \mathbf{\square}$, et $\bullet$. Les valeurs les plus fortes sont obtenues pour des bouées côtières dont les abords sont mal représentés dans un modèle global à $0.5^{\circ}$ de résolution.

Les erreurs moyennes au large sont généralement comprises entre 9 et $15 \%$ pour la hauteur significative $\mathrm{H}_{\mathrm{m} 0}$ (figure 4). Les erreurs les plus fortes avec le paramétrage de BIDLOT et al. (2005) sont concentrées dans la moitié ouest de l'Atlantique Nord et dans le Pacifique tropical, en particulier à l'est. Une amélioration importante au modèle a été apportée par le changement de paramétrage, qui représente la dissipation de la houle induite par le frottement à l'interface air-mer (ARDHUIN et al. 2008a) ce qui réduit les erreurs en zone tropicale (figure 5), sans dégrader les résultats par ailleurs (cf. figures 4 et 6). On remarque aussi la signature des courants équatoriaux, qui induisent des biais de l'ordre de $20 \mathrm{~cm}$ sur la hauteur significative car ils ne sont pas pris en compte à dans le système opérationnel actuel. La modification du paramétrage par moutonnement (ARDHUIN et al. 2008b) supprime la sensibilité de la mer du vent à la houle, ce qui permet de réduire les différences entre les partie est des bassins (moins de houle) et les parties ouest. Par rapport aux observations satellites, plus fiables que les bouées, la réduction de l'erreur est en moyenne de $1.7 \%$, passant 
de 13.1 à $11.4 \%$ en moyenne globale. Par rapport aux bouées la réduction est par exemple de $10.6 \%$ à $9.4 \%$ pour la hauteur $\mathrm{H}_{\mathrm{m} 0}$ à la bouée Brittany (figure 6 ) tandis que la fréquence moyenne $1 / T_{\mathrm{m} 02}$ est davantage biaisée ce qui augmente l'erreur de 9.7 à $10.6 \%$ pour cette bouée, alors que la tendance est plutôt à une réduction sur l'ensemble des bouées.
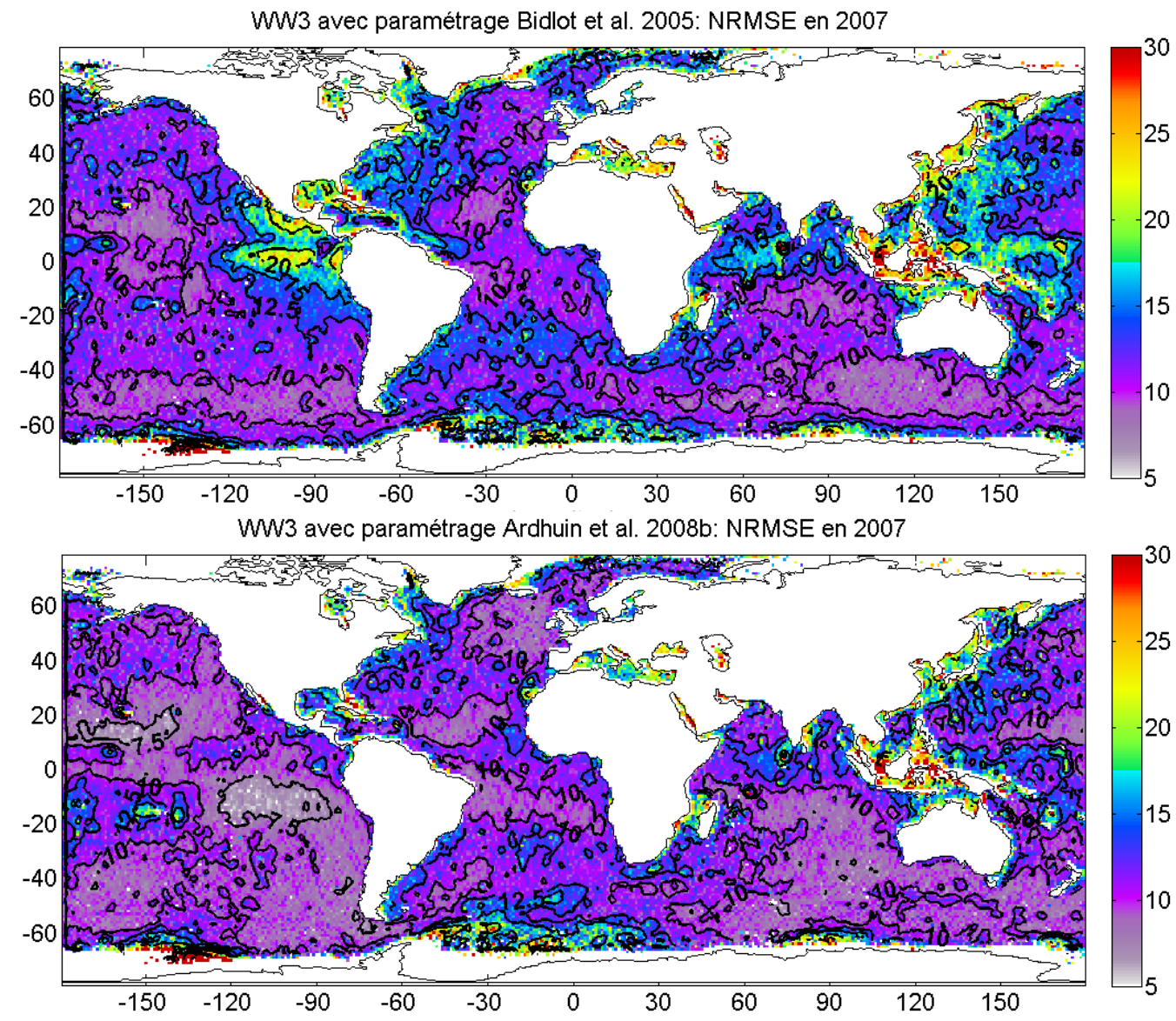

Figure 5: Carte des erreurs moyennes quadratiques normalisées par la valeur moyenne quadratique des observations dans chaque carreau de $1^{\circ}$ par $1^{\circ}$. Les observations utilisées combinent les altimètres en bande Ku des satellites GFO, JASON et ENVISAT pour l'année 2007 (voir RASCLE et al. 2008 pour la méthode). En haut : résultat avec le paramétrage de BIDLOT et al. (2005), en bas avec celui de ARDHUIN et al. (2008).

En Manche et Méditerranée, zones semi-côtières, les résultats sont moins bons avec des erreurs RMS normalisées de l'ordre de $20 \%$, pouvant atteindre $30 \%$ des analyses de $\mathrm{H}_{\mathrm{m} 0}$, voire même $40 \%$ en Adriatique. Un biais négatif systématique sur la hauteur $\mathrm{H}_{\mathrm{m} 0}$ est observé sur l'ensemble des bouées utilisées (ARDHUIN et al., 2007). Le tableau 1 présente les résultats obtenus sur l'année 2007 avec le 
paramétrage de BIDLOT et al. (2005). NRMSE représente l'erreur quadratique (RMSE) normalisée. Ces résultats, moins précis qu'en mer ouverte, sont expliqués en partie par les champs de vents de moins bonne qualité dans cette zone, caractérisée par une forte orographie, et par des systèmes météorologiques à dynamiques très rapides. Une calibration statistique des champs de vent devrait être réalisée afin d'améliorer leurs qualités et par conséquence celle des vagues.
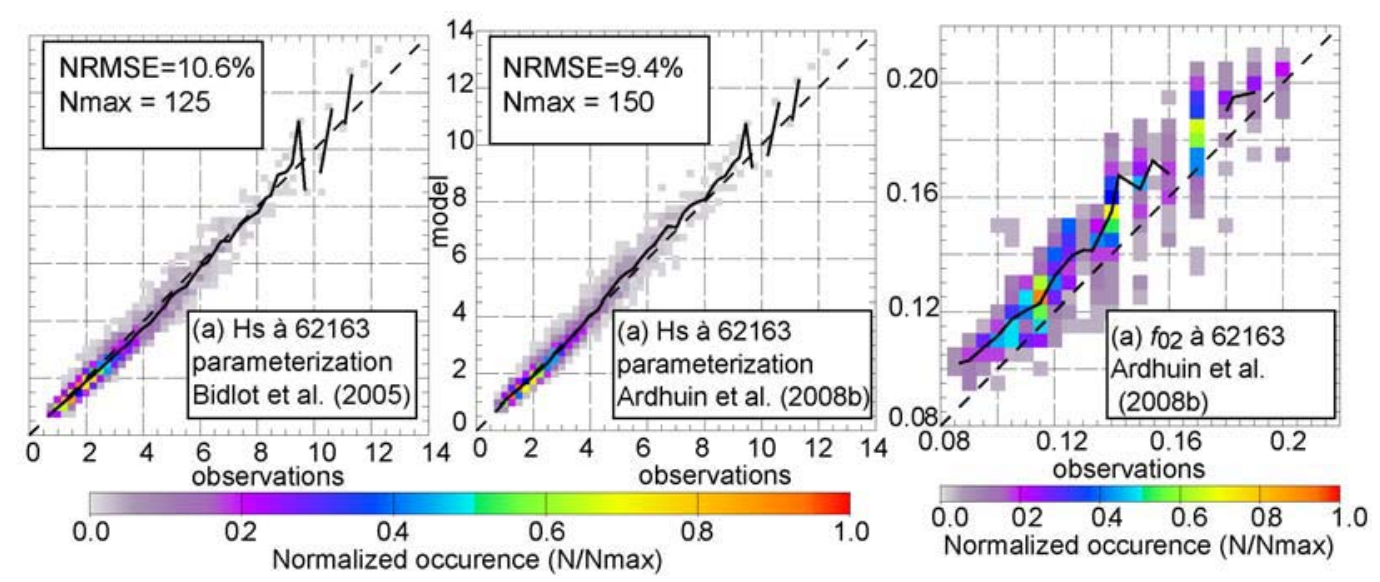

Figure 6: Observations et résultats du modèle sur l'année 2007 à la bouée Brittany (62163), au large de la Bretagne, pour la hauteur significative et le paramétrage ARDHUIN et al. (2008b), à gauche, celui de BIDLOT et al. (2005) au centre, et pour la fréquence moyenne $1 / T_{m 02}$ à droite avec le paramétrage de ARDHUIN et al. (2008b). Le trait pointillé matérialise la bissectrice, et le trait plein joint les maxima d'occurrence des valeurs modèle pour une valeur observée. Toutes les observations sont moyennées sur 3 heures.

Tableau 1. Erreurs sur la hauteur $H_{m 0}$ (année 2007) pour les bouées méditerranéennes françaises et espagnoles. Les numéros des bouées sont ceux de l'OMM.

\begin{tabular}{|c|c|c|c|c|}
\hline No de bouée (nom) $^{\circ}$ NRMSE(\%) & RMSE(m) & Biais(m) & r \\
\hline 61001 (DYFAMED) & 23.0 & 0.281 & -0.130 & 0.944 \\
\hline 61002 (Lion) & 17.8 & 0.340 & -0.070 & 0.959 \\
\hline 61196 (Begur) & 18.6 & 0.314 & -0.018 & 0.951 \\
\hline 61197 (Mahon) & 21.6 & 0.270 & -0.067 & 0.912 \\
\hline 61198 (Cabo de Gata) & 23.4 & 0.293 & -0.150 & 0.924 \\
\hline 61280 (Tarragona) & 25.5 & 0.281 & -0.183 & 0.928 \\
\hline 61281 (Valencia) & 25.5 & 0.274 & -0.154 & 0.910 \\
\hline 61417 (Cabo de Palos) & 22.1 & 0.266 & -0.138 & 0.925 \\
\hline 61430 (Dragonera) & 29.9 & 0.401 & -0.247 & 0.911 \\
\hline
\end{tabular}


L'évolution du système et la comparaison de ses performances avec les principaux centres de prévision météorlogiques est mise à jour tous les mois sur le site internet du JCOMM :

http://www.jcomm-services.org/Wave-Forecast-Verification-Project.html

\subsection{Modèles côtiers}

Par ailleurs, le SHOM collabore à de nombreuses campagnes scientifiques et a entrepris depuis 2003 un programme de mesure pour la validation des modèles côtiers. Les mesures réalisées en mer d'Iroise et devant la côte Aquitaine suggèrent qu'une part importante des erreurs côtières est liée à une précision insuffisante des spectres directionnels prévus au large. Lors de la campagne VIGICOTE-2004, entre mars et mai 2004, 3 bouées Datawell ont été déployées en partenariat avec le CETMEF : Blancs Sablons, Berthaume et Iroise (cf. figure 7).

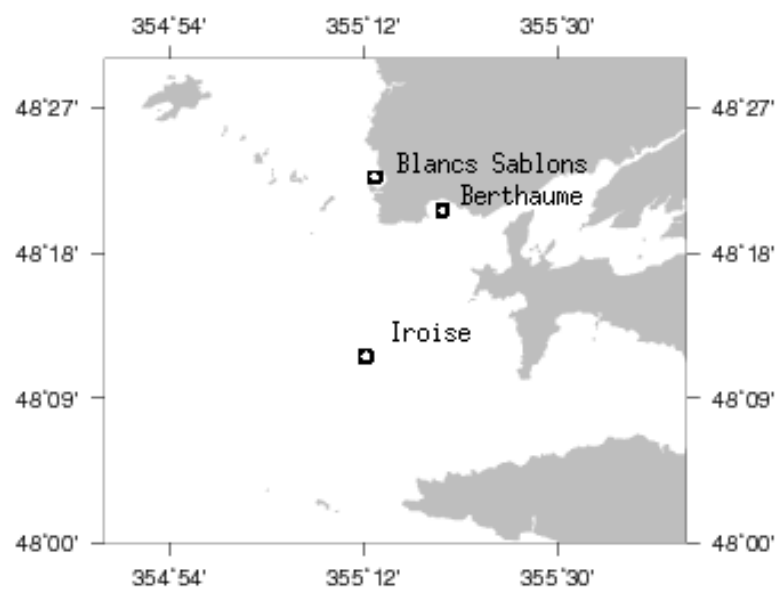

Figure 7 : Position des instruments pendant la campagne VIGICOTE2004.

Les positions des plages des Blancs Sablons et de Berthaume en font des endroits particulièrement intéressants pour étudier la performance des modèles côtiers. En effet, ces plages sont abritées des houles de sud-ouest et nord-ouest respectivement, avec un effet d'abri par l'archipel de Molène. Au contraire le point Iroise a une position assez dégagée, donnant la houle du large. A partir des spectres calculés à la position de la bouée 62052 («Beatrice», au large d'Ouessant), avec le modèle GASCOGNE à $0.1^{\circ}$ utilisant le paramétrage de BIDLOT et al. (2005), les erreurs obtenues par tracés de rayons à la côte sont plus importantes que celles des modèles hauturiers (cf. tableau 2).

Cette augmentation de l'erreur est en bonne partie liée à une mauvaise distribution angulaire de l'énergie au large d'une côte découpée (ARDHUIN, 2006). On a pu vérifier que les erreurs augmentaient moins à Oléron ou au Cap Ferret (campagnes 
de 2006 et 2007) où la côte est plus ouverte sur le large. Sur la côte du Finistère, pour une houle de période de $14 \mathrm{~s}$, une variation de l'étalement directionnel de $13^{\circ}$ à $38^{\circ}$ peut changer la hauteur significatuve de $25 \%$ aux Blancs Sablons et à Berthaume. Un effort sur la représentation directionnelle dans les modèles est nécessaire pour mieux prévoir les vagues à la côte. Cela demande aussi des observations directionnelles, qui font cruellement défaut sur les côtes françaises.

Tableau 2. Erreurs sur le $H_{m 0}$ pendant VIGICOTE2004.

\begin{tabular}{|c|c|c|c|}
\hline Instrument & NRMSE(\%) & RMSE(m) & Biais(m) \\
\hline Iroise & 18.6 & 0.35 & 0.005 \\
\hline Blancs Sablons & 26.2 & 0.37 & 0.16 \\
\hline Berthaume & 26 & 0.18 & 0.047 \\
\hline
\end{tabular}

\subsection{Ré-analyses et applications}

Outre la prévision en temps réel dans le cadre de Prévimer, nous avons réalisé un rejeu du modèle global avec une résolution de $1^{\circ}$ pour la période 2003-2007. Les résultats sont disponible par ftp ftp://ftp.ifremer.fr/ifremer/cersat/products/gridded/wavewatch3/HINDCAST .

Ce rejeu a été réalisé avec le paramétrage de BIDLOT et al. (2005) et validé par RASCLE et al., (2008). Un rejeu des domaines Gascogne et Méditerranée est en cours et sera disponible sur le même serveur. Un nouveau rejeu global avec les nouveaux paramétrages est disponible avec $0.5^{\circ}$ de résolution. Il est en cours de validation.

\section{$3 \quad$ Perspectives}

Les développements actuels portent sur la mise en place dans le code WAVEWATCH III d'une capacité de calcul des états de mer sur des maillages non structuré ou curvilignes, en collaboration avec le NOAA/NCEP et l'Université de Darmstadt (Allemagne), afin de remplacer les calculs par tracés de rayons dans les mers à marée. Une simple extension du code de tracés de rayon est également réalisée pour permettre un forçage spatialement variable aux frontières. La poursuite des travaux sur le paramétrage du déferlement (FILIPOT \& ARDHUIN 2008) devrait aussi améliorer les résultats, en particulier en présence de courant.

\section{$4 \quad$ Références bibliographiques}

1 ARDHUIN F. (2006). Quelles mesures pour la prévision des états de mer en zone côtière. Communications de l'Atelier Expérimentation et 
Instrumentation.

2 ARDHUIN F., BERTOLlI L., BIDLOT J.R., CAVALERI L., FILIPETTO V., LEFEVRE J.M., WITTMANN P. (2007) . Comparison of wind and wave measurements and models in the Western Mediterranean Sea. Ocean Engineering. Vol 34(3-4), pp. 526-541.

3 ARDHUIN F., MAGNE R. (2007). Scattering of surface gravity waves by bottom topography with a current. Journal of Fluid Mechanics. Vol. 576, pp. 235-264.

4 ARDHUIN F., CHAPRON B., COLLARD F. (2008). Strong decay of steep swells observed across oceans. Nature Geosciences, soumis.

5 ARDHUIN F., CHAPRON B., COLLARD F., QUEFFEULOU P., FILIPOT J.-F., HAMON M. (2008). Spectral wave dissipation based on observations: a global validation. Proceedings of the Chinese-German Joint Symposium, Darmstadt, Allemagne, août 2008.

6 BATTJES J.A., JANSSEN J.P.F.M. (1978). Energy loss and set-up due to breaking of random waves. Proceedings 16ème Int. Conf. of Coastal Eng., pp. 569-587, ASCE.

7 BIDLOT J., ABDALLA S. et JANSSEN P.A.E.M. (2005). A revised formulation for ocean wave dissipation in CY25R1, Memo. R60.9/JB/0516, Research Department, ECMWF, Reading, U. K.

8 BIDLOT J., LI J.G., WITTMANN P., FAUCHON M., CHEN H., LEFEVRE J., BRUNS T., GREENSLADE D., ARDHUIN F., KOHNO N., PARK S., GOMEZ. M.(2007). Inter-comparison of operational wave forecasting systems. Proceedings 10th Int. Workshop on Wave Hindcasting and Forecasting, Hawaii.

9 FILIPOT J.F., ARDHUIN F. (2008). Paramétrage du déferlement des vagues dans les modèles spectraux : approches semi-empiriques et physiques. Xèmes Journées Nationales Génie Civil - Génie Côtier, Sophia Antipolis.

10 KIRBY J.T. (1986). A general wave equation for waves over rippled beds. Journal of Fluid Mechanics. Vol. 162, pp 171-186.

11 RASCLE N., ARDHUIN F., QUEFFEULOU P., CROIZE-FILLON D. (2008). A global wave parameter database for geophysical applications. Part 1: wave-current-turbulence interaction parameters for the open ocean based on traditional parameterizations. Ocean modelling, sous presse.

12 TOLMAN H. (2007). The 2007 release of WAVEWATCH III, Proceedings 10th Int. Workshop on Wave Hindcasting and Forecasting, Hawaii. 